\title{
Pachyonychia Congenita Type PC-K6a: The first report in the Vietnamese population
}

\author{
Ha Thi Chu ${ }^{1}$, Doanh Huu Le ${ }^{2}$, Thieu Van Le ${ }^{3}$, Binh Bui Nguyen ${ }^{1}$, Janice Schwartz ${ }^{4}$, Quang Van Vu ${ }^{1,5}, *(\mathbb{0}$
}

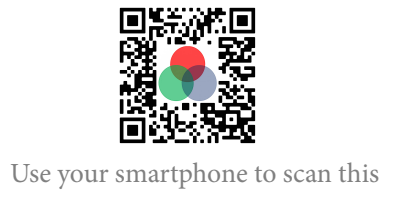

QR code and download this article

${ }^{1}$ Department of Pediatrics, Haiphong University of Medicine and Pharmacy, Haiphong, Vietnam

${ }^{2}$ National Hospital of Dermatology and Venereology, Hanoi, Vietnam

${ }^{3}$ Endoscopy and Exploration Functional Department, Viettiep friendship hospital, Haiphong, Vietnam

${ }^{4}$ Pachyonychia Congenita Project, Salt Lake City, Utah, USA

${ }^{5}$ Pediatric department, Green International Hospital, Haiphong, Vietnam

Correspondence

Quang Van Vu, Department of Pediatrics, Haiphong University of Medicine and Pharmacy, Haiphong, Vietnam

Pediatric department, Green International Hospital, Haiphong, Vietnam

Email: vvquang@hpmu.edu.vn

History

- Received: Mar 17, 2021

- Accepted: Jun 10, 2021

- Published: Jun 30, 2021

DOI : 10.15419/bmrat.v8i6.681

\section{Check for updates}

\section{Copyright}

( $)$ Biomedpress. This is an openaccess article distributed under the terms of the Creative Commons Attribution 4.0 International license.

ABSTRACT

Background: Pachyonychia congenita (PC) comprises a group of rare autosomal dominant genetic disorders. It is characterized by hypertrophic nail dystrophy, focal palmoplantar keratoderma, follicular keratosis, and oral leukokeratosis. It is associated with mutations in five differentiationspecific keratin genes: KRT6A, KRT6B, KRT6C, KRT16, or KRT17. The case is being reported for its rarity. To the best of our knowledge, this is the first report of PC, from Vietnam, confirmed by genetic analysis. Case presentation: A four-year-old Vietnamese girl presented with a thickened nail and oral leukokeratosis soon after birth. She was diagnosed with onychomycosis and chronic oral candidiasis and was treated with systemic anti-fungals in children's hospitals and dermatology departments multiple times; however, no treatments were effective. In collaboration with the International Pachyonychia Congenita Research Registry (IPCRR), the clinical features were consistent with a diagnosis of PC type PC-K6a. The genetic testing, performed through the IPCRR, shows a K6a N171K mutation. Conclusions: The IPCRR helps screen PC's clinical features and confirm a diagnosis at the molecular level, which is beneficial and crucial for validating the condition's clinical impression.

Key words: follicular papules, leukokeratosis, nail dystrophy, Pachyonychia Congenita

\section{INTRODUCTION}

Pachyonychia congenita (PC) is a rare autosomal dominant disorder of keratinization. It is characterized by hypertrophic nail dystrophy, follicular hyperkeratosis, painful plantar and palmar keratoderma, oral leukokeratosis, epidermal cysts, natal teeth, and hoarseness ${ }^{1}$. Genetic testing of PC is critical for diagnostic confirmation and genetic counseling ${ }^{1,2}$. The International Pachyonychia Congenita Research Registry (IPCRR, WCG IRB \#20040468), sponsored by Pachyonychia Congenita Project, was formulated in 2004 and since then has collected data on over 2,000 patients suspected of having PC and provides free genetic testing for those in the IPCRR. Research based on the IPCRR data has shown significant overlap between previous types of PC. Thus, a new classification was proposed in 2011 that grouped $\mathrm{PC}$ into five types: PC-K6a, PC-K6b, PC-K6c, PC-K16, and PC$\mathrm{K} 17$, which represents specific affected genes ${ }^{3}$. This is the classification now used to describe PC. Underlying keratin gene mutations have been described in the five keratin genes, KRT6A (OMIM 148041), KRT6B (OMIM 148042), KRT6C (OMIM 612315), KRT16 (OMIM 148067), and KRT17 (OMIM 148069), which alter keratins $6 a, 6 b, 6 c, 16$, and 17 , respectively ${ }^{4}$. These genes are expressed in the nail bed, palmoplantar epidermis, and mucosa. Keratins play a key role in epidermal cell integrity and mechanical strength, and mutations in any of these five keratin genes cause epidermolysis and compensatory hyperkeratosis at these sites $^{5}$.

Here, we report a case of this rare disease. To the best of our knowledge, this is the first PC case from Vietnam confirmed by genetic analysis.

\section{CASE PRESENTATION}

A four-year-old female patient was presented at our hospital (March 2016) because of leukokeratosis and thickened nails with a progressive course since early infancy. She is the first child of nonconsanguineous parentage with no history of genetic disease. She underwent an uncomplicated birth and all normal developmental milestones for her age. Her mother noticed nail changes at 15 days and the leukokeratotic plaques at 30 days. The child was diagnosed with onychomycosis, candida infection and was treated with local, systemic anti-fungals at pediatric and dermatology hospitals; however, all treatments were ineffective. A physical examination identified a distinct hypertrophic nail dystrophy of the feet and hands, with distal hyperkeratosis and curvature of nails especially on the fingers, which were wedge-shaped with yellowish discoloration (Figure 1A, C, D). Sometimes, the fingernails would become infected and fall off 

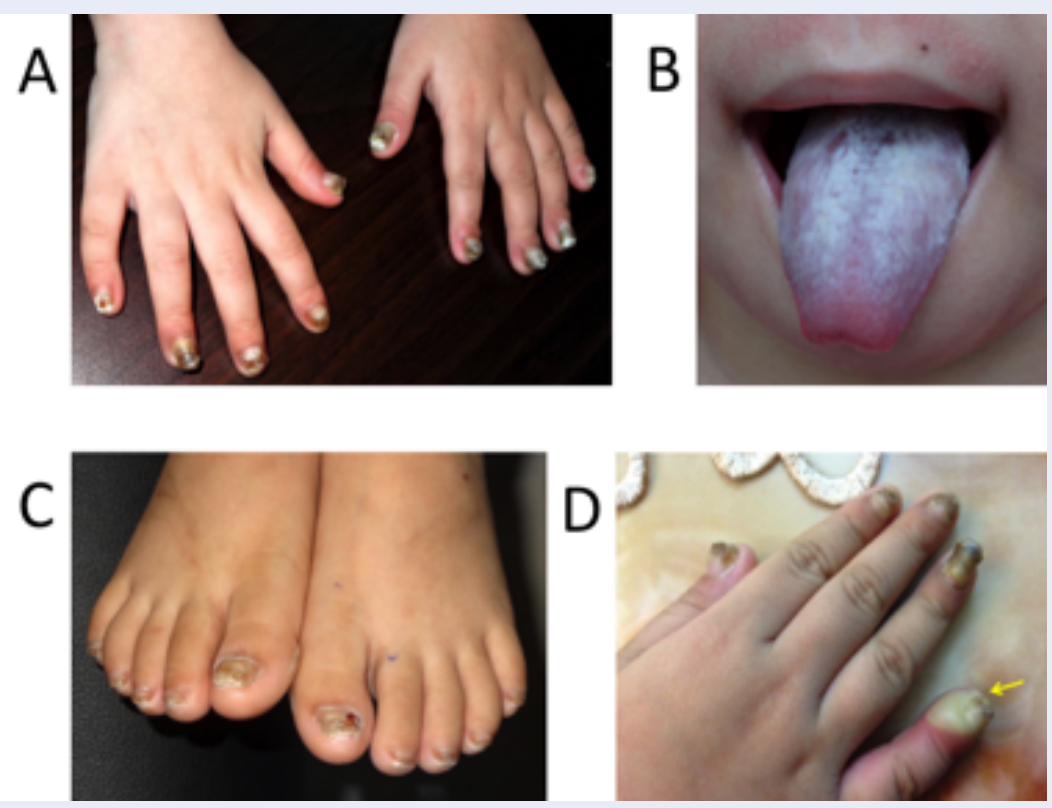

Figure 1: Nail dystrophy (A, all 10 fingernails thickened; C, all 10 toenails thickened; $D$, fingernails were infected); Oral leukokeratosis of the tongue was misdiagnosed initially as a candidal infection (B)

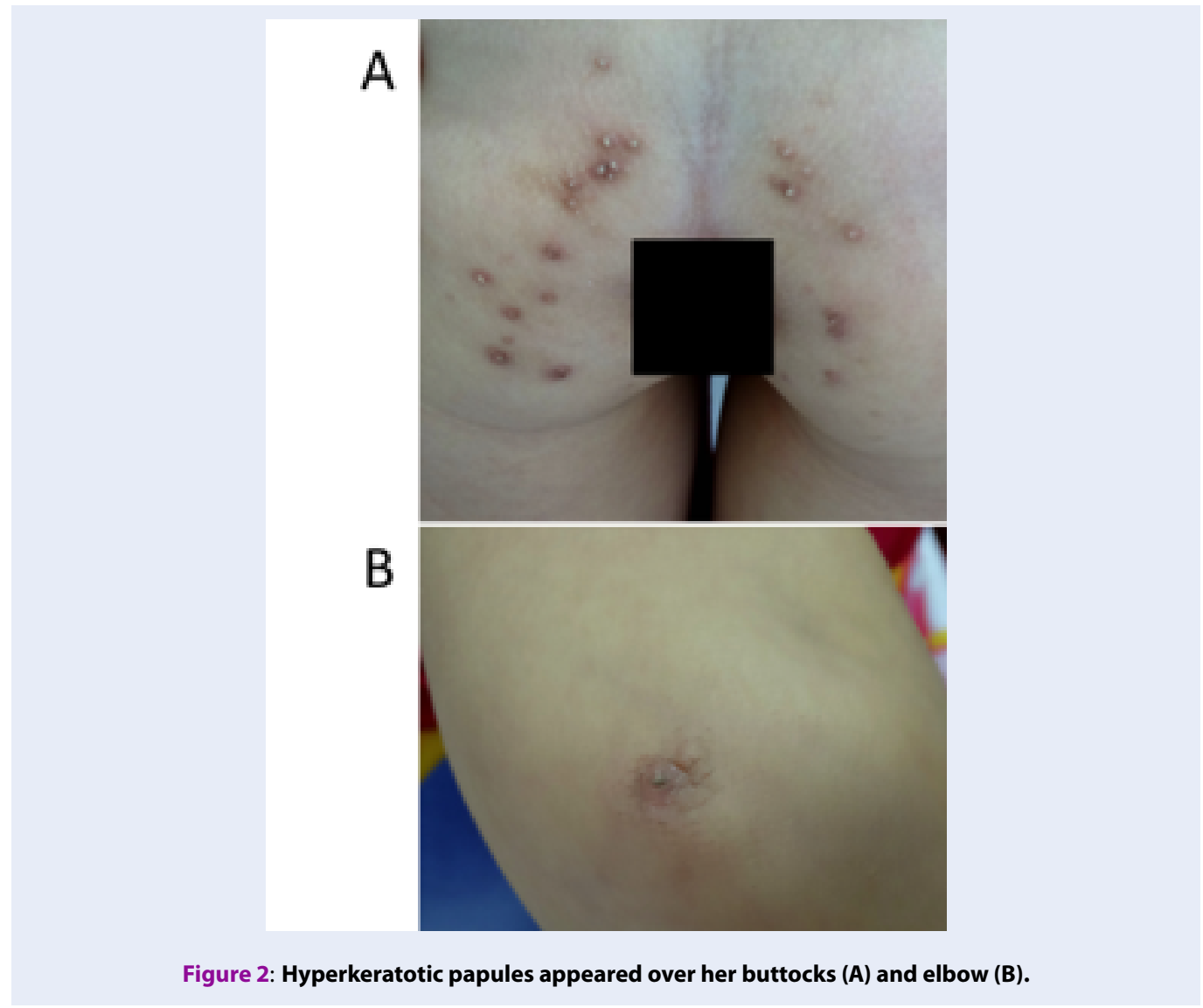


A

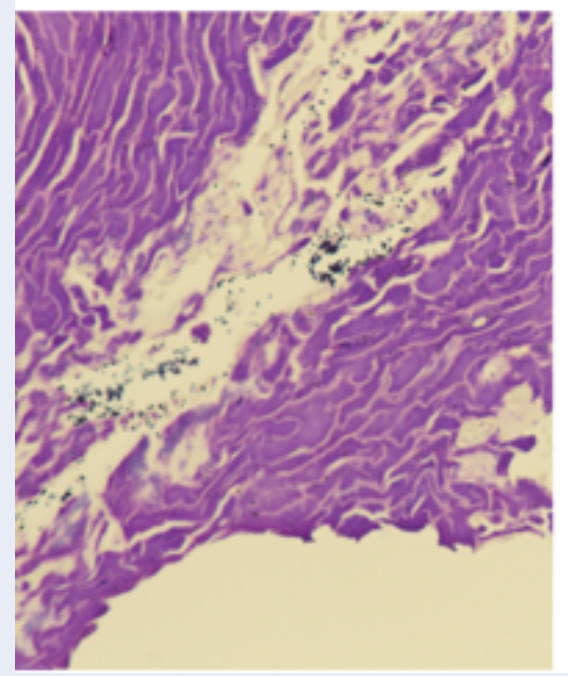

Figure 3: Histology of nail tissue showed marked proliferation of keratin (A) gram stain; (B) HE stain.
B

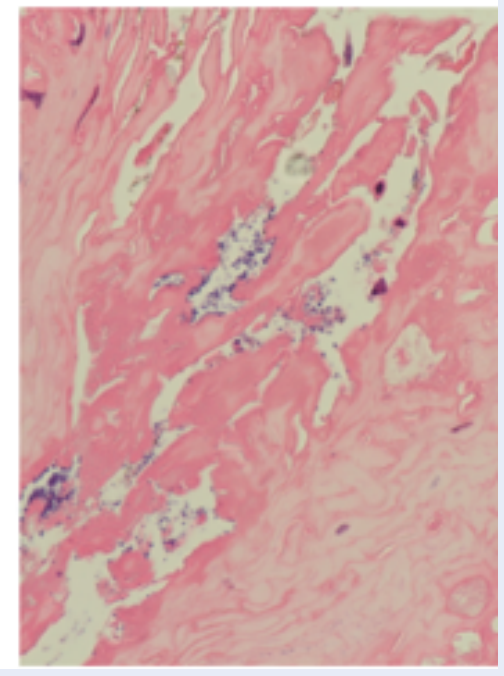

\section{Control: K6a, exon 1 Patient: K6a p.Asn171Lys}
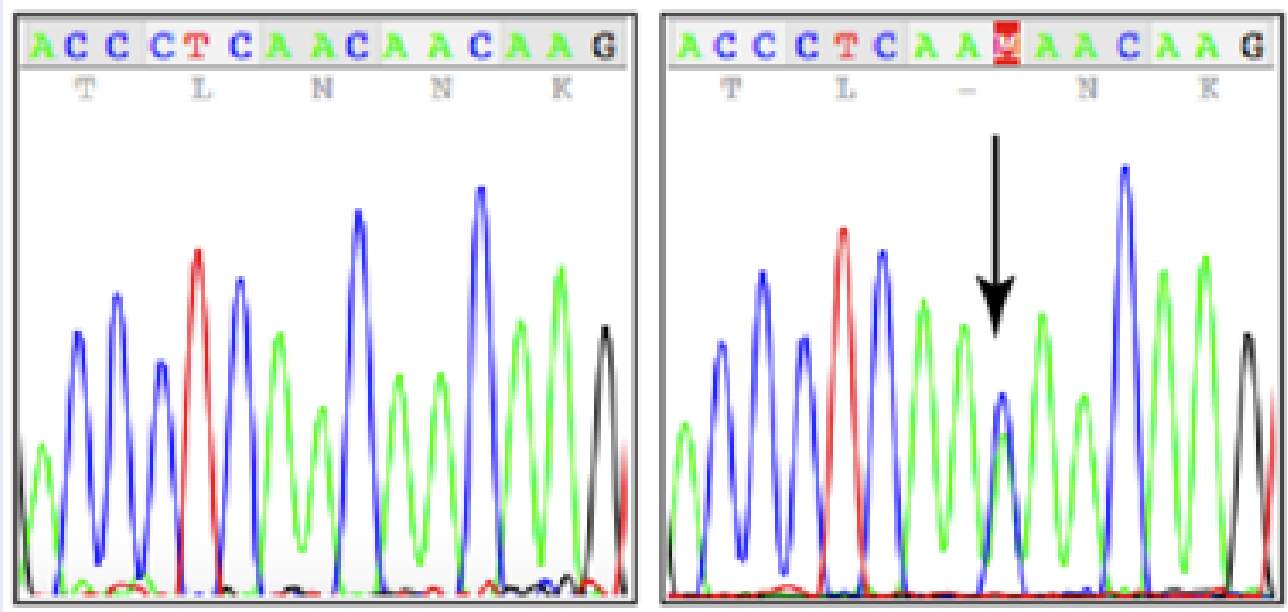

Figure 4: The K6a N171K mutation. Because of the mutation, one of the amino acid building blocks Asparagine (Asn or $\mathrm{N}$ ) is changed to Lysin (Lys or K); the correct protein is not produced. 
(Figure 1D). Oral leukokeratosis covered the entire dorsal aspect of her tongue and fluctuated with time (Figure 1B). She had focal plantar keratoderma, but at the time, it did not affect her walking or limit her mobility. The hoarseness of her voice was not reported. Hyperkeratotic papules appeared over her buttocks (Figure 2A) and elbows (Figure 2B). Hyperkeratotic papules were exacerbated by warm and dry weather. Topical keratolytics and emollients were prescribed but showed limited benefit. Routine laboratory investigations including complete hemogram, hepatic profile, and renal profile were within normal limits. Histologically, we identified the marked proliferation of keratin with an edematous change; no Aspergillus infection was observed in the nail specimens (Figure 3 A-B). This was also confirmed by immunohistochemical staining using an anti-Aspergillus antibody. No evidence of any malignancy was found during the comprehensive examination.

Based on the above findings, the patient was clinically diagnosed with PC. After being referred to the PC Project (www.pachyonychia.org), a saliva kit for genetic testing was freely provided by the PC Project. PC Project offers genetic testing to PC patients who enroll in the IPCRR (https://www.pachyo nychia.org/patient-registry/). Following the acquisition of informed consent according to the Declaration of Helsinki principles, genetic testing was performed to confirm the clinical diagnosis. The results revealed the KRT6A gene to have the recurring mutation at $\mathrm{N} 171 \mathrm{~K}$. Because of the mutation, one of the amino acid building blocks Asparagine (Asn or N) changed to Lysin (Lys or K); therefore, the correct protein is not produced.

After being diagnosed with PC, the patient was no longer mistreated. We suggested appropriate care and therapeutic solutions to improve her quality of life.

\section{DISCUSSION \& CONCLUSIONS}

Pachyonychia Congenita is a rare autosomal dominant keratin disorder caused by mutations in any of the five keratin genes: KRT6A, KRT6B, KRT6C, $K R T 16$, or $K R T 17^{6}$. The major phenotypical features of PC reported in more than $90 \%$ of patients with this condition are nail dystrophy, plantar keratoderma, and plantar pain. Other common clinical findings of PC include oral leukokeratosis, follicular hyperkeratosis, and the formation of cysts ${ }^{1,7}$. Additionally, natal teeth, a phenomenon of erupted teeth present at birth, have also been reported in a few patients with PC-K17. Based on clinical characteristics, PC was classified into two primary subtypes: the JadassohnLewandowski PC type 1 (mutations in KRT $6 A$ and
KRT16) and Jackson-Lawler PC type 2 (mutations in KRT6B and KRT 17). Because of a significant phenotype overlap between cases previously classified as PC types 1 and 2, a new classification divided PC into four subtypes based on the specific keratin defect ${ }^{1,6,7}$ and later into five subtypes. $\mathrm{PC}$ subtypes are now classified as PC-K6a, PC-K6b, PC-K6c, PC-K16, and PC$\mathrm{K} 17$ for mutations in KRT6A, KRT6B, KRT6C, KRT16, and KRT17 genes, respectively. Due to the overlapping clinical features between the different subtypes of PC, genetic testing is required to confirm the diagnosis $^{2}$. The patient we herein report showed typical manifestations including thickened nails, oral leukocytosis, hyperkeratotic papules, and focal plantar keratoderma soon after birth. However, the diagnosis was missed and delayed until the age of four. Her nail dystrophy and oral leukokeratosis were diagnosed as onychomycosis and chronic candidiasis, respectively. This misdiagnosis can be explained by the following reasons: there is insufficient awareness of this rare disease, and Vietnam is a tropical country, where infectious diseases including fungal diseases are common ${ }^{8,9}$. In our hospital, the patient also was first suspected of having onychomycosis. The nail culture also showed Aspergillus versicolor; however, was this fungus pathogenesis or merely superinfection? Histologically, no finding of Aspergillus versicolor in the nail specimens examined was seen but there was marked proliferation of keratin. No evidence of malignancy was observed either. Therefore, a diagnosis of PC was made. Genetic testing was performed that revealed the K6a N171K mutation, thereby confirming the clinical diagnosis. This mutation has been previously reported in 18 individuals in the world ${ }^{10}$. Our patient represents one of more than $45 \%$ of PC cases that appear spontaneously with no family history (de novo mutation).

According to data of PC project, there were 977 PC individuals in 517 families worldwide confirmed by genetic analysis; 115 mutations across the five keratin genes were identified. The percentage of KRT6A, KRT6B, KRT6C, KRT16, and KRT17 gene mutations is $39,9,3,33$, and 16 , respectively. PC-K6a affects the largest group of PC patients characterized by earlyonset and extensive nail dystrophy; severe, painful plantar keratoderma; follicular hyperkeratosis; and oral leukokeratosis $^{10,11}$. While nearly $100 \%$ of PCK6a patients over six years old have painful plantar keratoderma that negatively impacts quality of life, our patient seldom has pain. This may be explained by her young age. After the confirmed diagnosis, the patient is no longer mistreated. Although there is no specific treatment for $\mathrm{PC}$, the supportive therapies 
available on the PC Project website have proven effective to improve the daily activities of our patient. We continue to support the patient to improve her quality of life. The experience from this first PC case has helped us provide an early diagnose to many other PC patients scattered throughout Vietnam. In summary, we report the first case of PC-K6a confirmed by genetic analysis from Vietnam under the support of the PC Project. This study stressed the importance of genetic testing to confirm the diagnosis and genetic counseling.

\section{ABBREVIATIONS}

PC: Pachyonychia congenita

IPCRR: International Pachyonychia Congenita Research Registry

\section{ACKNOWLEDGMENTS}

The authors would like to thank the patient and her family in this study for their cooperation. Thanks also to PC project including Holly Evans for her help with data collection to the International Pachyonychia Congenita Consortium Genetics Team for useful suppor.

\section{AUTHOR'S CONTRIBUTIONS}

HTC, QVV, DHL, TVL and BBN participated in the study design, protocol development and performance, data analysis, interpretation of data, and writing of the manuscript and carried out the clinical data collection and data analysis. QVV and JS reviewed and revised the manuscript, making important intellectual contributions. All authors read and approved the final manuscript.

\section{FUNDING}

Genetic testing work was supported by Pachyonychia Congenita Project.

\section{AVAILABILITY OF DATA AND MATERIALS}

Data and materials used and/or analysed during the current study are available from the corresponding author on reasionable request.

\section{ETHICS APPROVAL AND CONSENT TO PARTICIPATE}

This study was conducted in accordance with the amended Declaration of Helsinki. Written informed consent was obtained from the patient and his parents for publication of these data and for the accompanying images.

\section{CONSENT FOR PUBLICATION}

Not applicable.

\section{COMPETING INTERESTS}

The authors declare that they have no competing interests.

\section{REFERENCES}

1. Eliason MJ, Leachman SA, Feng BJ, Schwartz ME, Hansen CD. A review of the clinical phenotype of 254 patients with genetically confirmed pachyonychia congenita. J Am Acad Dermatol. 2012;67(4):680-6. PMID: 22264670. Available from: 10.1016/j.jaad.2011.12.009.

2. Rusu C, Murgu A, Chiriac AE, Wilson NJ, Smith FJD. First Report of Pachyonychia Congenita Type PC-K6a in the Romanian Population. Maedica (Buchar). 2017;12(2):123-6. PMID: 29090033.

3. McLean WH, Hansen CD, Eliason MJ, Smith FJ. The phenotypic and molecular genetic features of pachyonychia congenita. J Invest Dermatol. 2011;131(5):1015-7. PMID: 21430705. Available from: 10.1038/jid.2011.59.

4. Pachyonychia Congenita - GeneReviews ${ }^{\circ}$ - NCBI Bookshelf [Internet]. [cited 2018 Aug 22]. Available from: https://www.ncb i.nlm.nih.gov/books/NBK1280/;

5. Shah S, Boen M, Kenner-Bell B, Schwartz M, Rademaker A, Paller AS. Pachyonychia congenita in pediatric patients: natural history, features, and impact. JAMA Dermatol. 2014;150(2):146-53. PMID: 24132595. Available from: 10. 1001/jamadermatol.2013.6448.

6. Wilson NJ, Leachman SA, Hansen CD, McMullan AC, Milstone LM, Schwartz ME. A large mutational study in pachyonychia congenita. J Invest Dermatol. 2011;131(5):1018-24. PMID: 21326300. Available from: 10.1038/jid.2011.20.

7. Ghazawi FM, Hassani-Ardakani K, Henriques L, Jafarian F. Identification of a novel substitution mutation (R103C) in the rod domain of the keratin 17 gene associated with pachyonychia congenita type 2. Int J Dermatol. 2018;58(2):233-236. PMID: 29904921. Available from: 10.1111/ijd.14082.

8. Vu QV. Acne fulminans in a Vietnamese boy successfully treated with prednisolone and ibuprofen: A case report. Biomed Res Ther. 2018;5(9):2708-2711. Available from: 10. 15419/bmrat.v5i9.483.

9. Tran TT, Vu QV, Wada T, Yachie A, Minh HLT, Nguyen SN. Novel HAX1 Gene Mutation in a Vietnamese Boy with Severe Congenital Neutropenia. Case Rep Pediatr. 2018;2018:2798621. PMID: 30598852. Available from: 10.1155/2018/2798621.

10. PC Data [Internet]. [cited 2021 Feb 26]. Available from: https:/ /www.pachyonychia.org/pc-data/;.

11. Forrest CE, Casey G, Mordaunt DA, Thompson EM, Gordon L. Pachyonychia Congenita: A Spectrum of KRT6a Mutations in Australian Patients. Pediatr Dermatol. 2016;33(3):337-42. PMID: 27041546. Available from: 10.1111/pde.12841. 